\title{
Toughening induced by the formation of facets in mode I+III brittle fracture: experiments versus a two-scale Cohesive Zone model
}

\author{
M.L. Hattali ${ }^{2}$, T. Cambonie ${ }^{3}$, V. Lazarus ${ }^{1}$ \\ ${ }^{1}$ IMSIA, CNRS, EDF, CEA, ENSTA Paris, Institut Polytechnique de Paris, 828 bd des Maréchaux, 91762 Palaiseau cedex, France \\ ${ }^{2}$ Université Paris-Saclay, CNRS, FAST, Orsay, France \\ ${ }^{3}$ LTDS-ENTPE : Laboratoire de Tribologie et Dynamique des Systèmes - Ecole Nationale des Travaux Publiques d'Etat
}

\begin{abstract}
When subjected to some anti-plane shear mode III loading, segmentation of the crack front frequently occurs during propagation: even if the crack is initially planar, propagation produces facets/segments rotated toward the shear free direction. Here, we examine, both experimentally and theoretically, the effect of this microstructure on the effective macroscale brittle fracture toughness. Experiments performed on PMMA beams reveal that the critical load leading to abrupt rupture increases with mode III to mode I ratio. This apparent macroscopic toughening is usually taken into account by invoking a specific mode III toughness in addition to the mode I one. By applying thoroughfully a micro/macroscale Cohesive Zone $(\mathrm{CZ})$ model that we have recently developed, we demonstrate that an additional material constant is useless here since this toughness increase can be attributed mainly to the presence of the facets at the microscale, whose geometry can be anticipated to depend on the classical mode I material constants. More precisely, two related physical mechanisms are generated due to the formation of a disconnected crack front: (i) changes in fractured surface area in comparison to a straight propagation, and (ii) crack shielding caused by the facets that reduce the effective crack opening. While the first effect is obvious to quantify, we show that the second plays an essential role but is more complex to take into account: it depends on the solution of the three-dimensional elasticity problem in presence of the facets, that is considered in the CZ model. We illustrate on the experiments how to use this approach in practice to determine the critical fracture threshold.
\end{abstract}

Keywords: Brittle fracture, Linear Elastic Fracture Mechanics, Mode I+III, Tilted facets, Factory roof pattern, Effective fracture toughness

Working towards the sustainability of materials is an undeniable, albeit indirect, way to reduce our carbon footprint. Lifetime predictions of industrial components (aircraft, power-plants, railways...) are often greatly underestimated. While usually safe, too frequent replacements of components is a waste of energy and material. One source of the underestimation comes from simplifications made in the actual models, especially on the geometry of the crack (Lazarus, 2003, 2011). Here, we focus on the study of surface topology effect, generated by mode I+III loading conditions, on crack propagation. In this case, appearance of inclined facets within fracture surfaces demonstrates the need for a 3D picture.

Patterns on broken surfaces are well-known from everyday experience, but surprisingly, how and why they form are wide-open questions, notably when a pre-existing crack or fault is subjected to some anti-plane shear/torsion mode III loading. This situation occurs in engineering: turbines (Fremy et al., 2014), wind power plant, railway (Bonniot et al., 2018), but also in the Nature: stick-slip earthquake (Cambonie et al., 2019), bone fracture. Segmentation of the crack front then frequently occurs during propagation: Even if the crack is initially planar with a straight front, both the crack front and surfaces become distorted as propagation occurs, when some facets (also known as segments or echelons) orientated toward the shear free direction appear and further coalesce during propagation (Palaniswamy and Knauss, 1975; Hull, 1995; Lazarus et al., 2008). First disconnected, the ligaments between the facets are broken in a second step. An example of the formation of those facets, their further coalescence and a postmortem picture of the fracture surface are given in Fig. 1. 


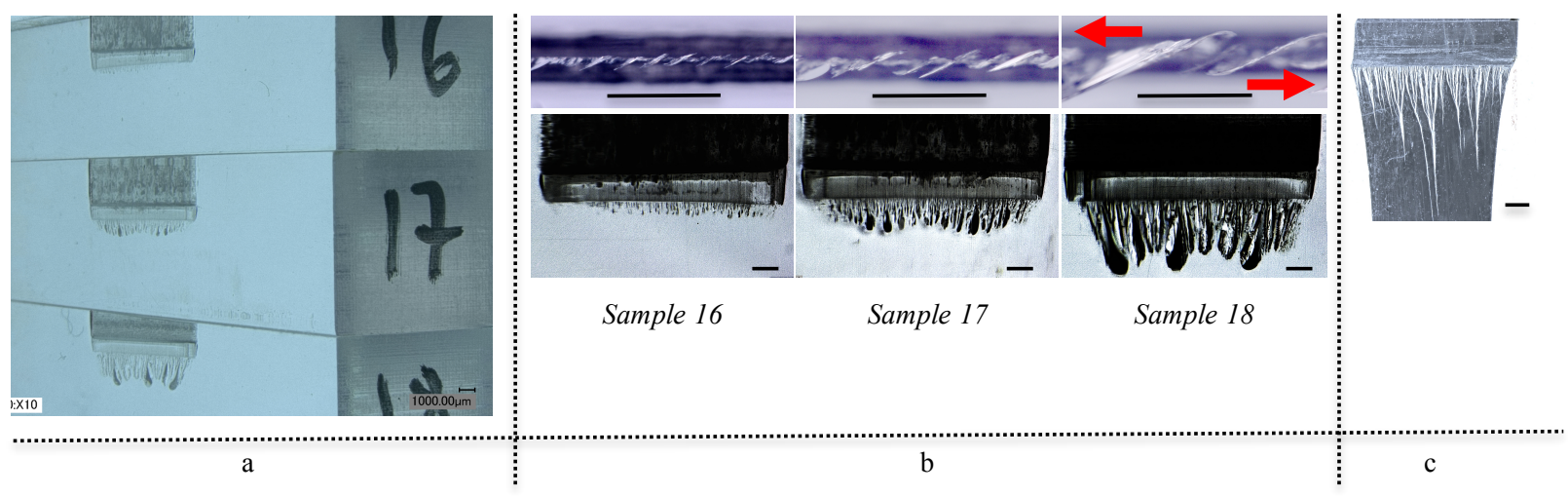

Figure 1: Facets formation and coalescence as observed by transparency in fatigue bending experiments performed on PMMA (Chen et al., 2015; Cambonie et al., 2019; Lazarus et al., 2020). Columns a, b : In-situ pictures of the facets in three different samples (named 16, 17, 18) observed at increasing stages of the propagation outlining the facet apparition and further coalescence: (a) Perspective view; (b) Bottom (first row) and front views (second row). Column c: postmortem picture of the fracture facies; the black surface corresponds to the fracture surface left by the facets and the white lines to the ultimate fracture of the ligaments between them. The bar scales are all $1 \mathrm{~mm}$.

This phenomenon appears (i) in a large set of materials: metals (Eberlein et al., 2017), polymers (Lazarus et al., 2008; Lin et al., 2010), glass (Sommer, 1969), cheese (Goldstein and Osipenko, 2012), soft matter (Ronsin et al., 2014), rocks (Pollard et al., 1982), tectonic plates (Cambonie et al., 2019); and (ii) from the scale of metallic grain boundaries (Qiao and Argon, 2003; Lambert-Perlade et al., 2004; Andrieu, 2013) to the kilometer geological scales (Pollard et al., 1982) through the lab (Lazarus et al., 2008) and engineering structures scales (Frémy, 2012). It occurs both in fatigue (Lazarus et al., 2008), under cyclic loading, and above the brittle fracture threshold (Goldstein and Osipenko, 2012; Lin et al., 2010; Pham and Ravi-Chandar, 2016). As in the experiments of Fig. 1, the facets generally coalescence during propagation by screening of every other facet (Pons and Karma, 2010; Chen et al., 2015; Pham and Ravi-Chandar, 2017) with a few exceptions (Sommer, 1969; Wu et al., 2007).

Even if initially planar with a straight front, why does the crack follow such a tortuous path instead of a simple straight and planar one once mode III loading is applied? The debate is not completely over, and it will not be the topic of this paper, but basically it is linked to a blend of (i) energy minimisation principles that favor the formation of tilted facets rotated toward the shear free direction (Lazarus et al., 2001a,b; Hull, 1995; Pollard et al., 1982), triggered by (ii) inevitable imperfections that act as spots where the nucleation and the segmentation instability initiate (Pons and Karma, 2010; Leblond et al., 2011; Leblond and Lazarus, 2015; Chen et al., 2015; Pham and Ravi-Chandar, 2017; Doitrand and Leguillon, 2018; Leblond et al., 2019; Vasudevan et al., 2020).

Toughening during mixed mode fracture is a common observation. For instance, an increase of fracture resistance with the amount of mode II versus mode I is generally reported for mode I+II interfacial crack propagation (Cao and Evans, 1989). In this case, in addition to plastic and viscoelasticity dissipations, reduction of the sliding crack opening caused by the contact zones along the unavoidable rough interface has been evidenced to play an important role (Liechti and Chai, 1992; Zebar et al., 2020). This last effect, called crack shielding in Evans and Hutchinson (1989) reduces the apparent energy release rate. Similarly, toughening induced by the presence of mode III has been reported in several experiments (Davenport and Smith, 1993; Liu et al., 2004; Lin et al., 2010; Eberlein et al., 2017) and may be related to the propagation of the disconnected facets. To test the pertinence of this correlation, the apparent macroscopic energy release rate has to be related to the microscale of the facets. This is precisely the aim of a twoscale Cohesive Zone (CZ) approach that we have recently developed (Leblond et al., 2015; Lazarus et al., 2020). Comparing this approach with 4 points bending experiments, we demonstrate that the toughening due to the presence of mode III can be attributed to the sole change of geometry induced by the facets without invoking (Lin et al., 2010; Leblond et al., 2019) a specific mode III fracture toughness in addition to the mode I value. The shape of the facets comes in through two ways: modification of the fracture surface area and crack shielding. The last is both similar to the shielding described for mode I+II in Evans and Hutchinson (1989), in the sense that out-of-plane asperities are 
responsible of the reduction of the crack opening in either case, but also different in the sense that the decrease of the opening is induced by the unbroken ligaments between the facets in mode I+III and by contact zones between the surface aspirities in mode I+II.

While the change in surface area is easy to take into account, the crack shielding induced by the facets in mixed mode I+III calls for a three-dimensional approach. It can be studied by the following two-scale micro/macro view. Assume, as usually observed, that facets appear at a small scale along the crack front. At a larger scale, once they have emerged, the crack advance corresponds to the propagation of the most advanced tips of these segments. By de-zooming sufficiently, the picture is the one of a coplanar equivalent extension, encompassing the facets, with a straight front connecting their most advanced points. Due to partial breaking of the material, the opening of this effective front and its Energy Release Rate (ERR) for a given loading, are lower than if the extension was smooth and coplanar. Consequently, it yields an increase of the apparent brittle fracture toughness at the macroscale, that we aim to bring out in this paper by interpreting some new experimental results in the light of a recent Cohesive Zone two-scale approach (Leblond et al., 2015; Lazarus et al., 2020).

The main ideas and equations of this model are recalled in Section 1. The experiments, performed using a 4 points bending setup and Poly(methyl methacrylate) polymer (PMMA) samples are presented in Section 2. We measure the crack geometry on the broken samples following the procedure described in Cambonie and Lazarus (2014) and use the datas as inputs in the $\mathrm{CZ}$ model. In this way, we obtain a critical loading in agreement with the experiments (Section 3). This demonstrates that the increase of the fracture threshold observed experimentally (i) can be attributed to the apparition of facets that require higher load to propagate and (ii) can be evaluated by the $\mathrm{CZ}$ model from the knowledge of the facet geometry. This roots the understanding of the observed toughening at the microscale and shifts the difficulty to the determination of the facet geometry. In Section 4, some ways to determine this geometry and the main physical mechanisms involved in the toughening are discussed.

\section{Cohesive Zone model}

\subsection{Principle of the method}

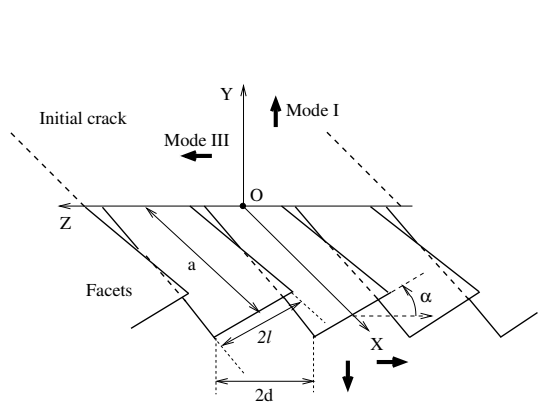

(a) $3 \mathrm{D}$ view before any coalescence

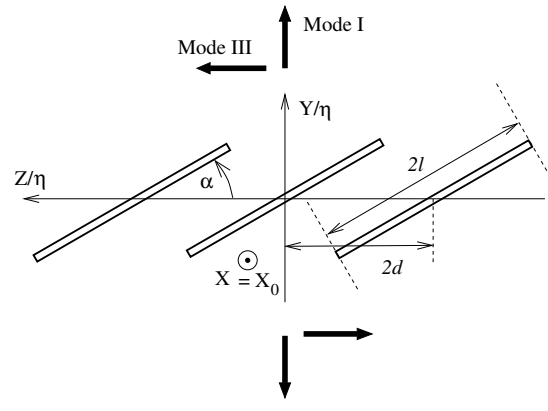

(b) Internal problem at the microscale

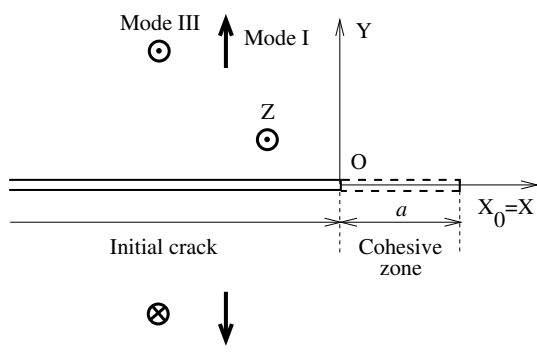

(c) External problem at the macroscale

Figure 2: Sketch and notations of the initial crack and the facet formation: decomposition of the initial 3D problem (a) in two 2D problems: at the microscale (obtained by rescaling $X$ and $Y$ axis by $X / \eta$ and $Y / \eta$, with $\eta \equiv d / a \rightarrow 0$ ), the facets appear as an array of parallel echelon cracks subjected to remote stresses (b); at the macroscale, they are embedded in a cohesive zone (c).

In presence of mode III, some facets (Fig. 1), nucleate along the crack front at the microscale. This changes the effective loading that makes them advance deeper into the material. The idea of the model developed by Leblond et al. (2015) in the framework of Linear Elastic Fracture Mechanics, is to embed the facets in a Cohesive Zone at the macroscale, its advance being ruled by some effective loading at its tip, that depends on the geometry of facets. Assuming that the spacing between the facets is small in comparison to their length, i.e. that $\eta \equiv d / a \ll 1$, a closed form solution can be found using matched asymptotic expansions. 
More precisely, consider a planar and straight crack subjected to mode I+III remote loading yielding uniform Stress Intensity Factors (SIF) $K_{I}, K_{I I I}$ along its front (Fig. 2a). When $\eta \rightarrow 0$, the facets can be considered as a bi-dimensional periodic array of tilted cracks at the microscale (internal problem, Fig. 2b) and as a growing CZ at the macroscale (external problem, Fig. 2c) whose propagation is ruled by the effective SIF $K_{I}^{e f f}, K_{I I}^{e f f}$ at the tip of the Cohesive Zone. Notice that this tip corresponds to the position of the line connecting the position of the most advanced points of the facets, so that its propagation corresponds in fact to the growth of the facets in the $X$ direction. The picture at the macroscale is obtained by zooming out, the facets then appear to be encompassed in the cohesive zone. Details of the method are complex and given in Leblond et al. (2015). Here we give only a rough picture of the method and recall the equations necessary for the determination of $K_{I}^{e f f}, K_{I I I}^{e f f}$, the dimensionless input parameters being the Poisson ratio $v$, the geometry of the facets $(\eta, \ell / d, \alpha)$ and the initial mode mixity ratio $K_{I I I} / K_{I}$.

\subsection{Determination of $K_{I}^{e f f}, K_{I I I}^{e f f}$}

The opening of the cohesive zone would be larger if it was completely broken than partially by facets. This explains why the effect of the facets appear in the matched asymptotic expansions as some additional surface tractions $\pm\left[p(X) \mathbf{e}_{Y}+q(X) \mathbf{e}_{Z}\right]$ exerted on the faces of the CZ. These additional pinning forces decrease the effective loading at the crack front and yield lower than nominal SIF at the crack tip of the cohesive zone:

$$
\left\{\begin{array}{l}
K_{I}^{e f f}=\sqrt{\frac{2}{\pi}} \int_{0}^{a} p(X) \frac{\mathrm{d} X}{\sqrt{a-X}} \\
K_{I I I}^{e f f}=\sqrt{\frac{2}{\pi}} \int_{0}^{a} q(X) \frac{\mathrm{d} X}{\sqrt{a-X}} .
\end{array}\right.
$$

The corresponding energy release rate can be obtained using Irwin (1958)'s formula:

$$
G=\frac{1-v^{2}}{E}\left(K_{I}^{2}+\frac{1}{1-v} K_{I I I}^{2}\right) \text { and } G_{e f f}=\frac{1-v^{2}}{E}\left(\left(K_{I}^{e f f}\right)^{2}+\frac{1}{1-v}\left(K_{I I I}^{e f f}\right)^{2}\right) .
$$

In Fig. 2a, the facets are sketched before any possible coalescence and the notations $a, \ell, d, \alpha$ describing their geometry are introduced. Afterwards, two situations may arise: (i) The facets propagates keeping $d$ constant; (ii) The facets progressively coalesce (as in Fig. 1), implying that the distance $d$ between them increases with $a$. Assuming that $\alpha$ and $\ell / d$ are constant along the facets, the additional surface tractions $p(X)$ and $q(X)$ on the $\mathrm{CZ}$ (for $X \in[0, a]$ ) can be obtained by a set of equations, that depends whether coalescence has occurred or not:

- Without coalescence, $d$ is independent of $X, p(X)$ and $q(X)$ for $X \in[0, a]$ can be obtained by solving the following equations:

$$
\left\{\begin{array}{c}
\left(\mathcal{A}_{22}+2 v \mathcal{A}_{12}\right) \frac{\mathrm{d} p}{\mathrm{~d} X}-\mathcal{A}_{23} \frac{\mathrm{d} q}{\mathrm{~d} X}+\frac{1}{\pi \eta a} P V \int_{0}^{a} p\left(X^{\prime}\right) \sqrt{\frac{a-X^{\prime}}{a-X}} \frac{\mathrm{d} X^{\prime}}{X^{\prime}-X} \\
=\frac{-\left(\mathcal{A}_{22}+2 v \mathcal{A}_{12}\right) K_{I}+\mathcal{A}_{23} K_{I I I}}{2 \sqrt{2 \pi} X^{3 / 2}} \\
-\left(\mathcal{A}_{23}+2 v \mathcal{A}_{13}\right) \frac{\mathrm{d} p}{\mathrm{~d} X}+\mathcal{A}_{33} \frac{\mathrm{d} q}{\mathrm{~d} X}+\frac{1}{\pi(1-v) \eta a} P V \int_{0}^{a} q\left(X^{\prime}\right) \sqrt{\frac{a-X^{\prime}}{a-X}} \frac{\mathrm{d} X^{\prime}}{X^{\prime}-X} \\
=\frac{\left(\mathcal{A}_{23}+2 v \mathcal{A}_{13}\right) K_{I}-\mathcal{A}_{33} K_{I I I}}{2 \sqrt{2 \pi} X^{3 / 2}} .
\end{array}\right.
$$


- With coalescence, that we assume to be at a constant rate, $d / a=\eta$ whatever $X$, the equations become:

$$
\left\{\begin{array}{c}
\left(\mathcal{A}_{22}+2 v \mathcal{A}_{12}\right)\left(\frac{\mathrm{d} p}{\mathrm{~d} X}+\frac{p}{X}\right)-\mathcal{A}_{23}\left(\frac{\mathrm{d} q}{\mathrm{~d} X}+\frac{q}{X}\right) \\
+\frac{1}{\pi \eta X} P V \int_{0}^{a} p\left(X^{\prime}\right) \sqrt{\frac{a-X^{\prime}}{a-X}} \frac{\mathrm{d} X^{\prime}}{X^{\prime}-X} \\
=\frac{\left(\mathcal{A}_{22}+2 v \mathcal{A}_{12}\right) K_{I}-\mathcal{A}_{23} K_{I I I}}{2 \sqrt{2 \pi} X^{3 / 2}} \\
-\left(\mathcal{A}_{23}+2 v \mathcal{A}_{13}\right)\left(\frac{\mathrm{d} p}{\mathrm{~d} X}+\frac{p}{X}\right)+\mathcal{A}_{33}\left(\frac{\mathrm{d} q}{\mathrm{~d} X}+\frac{q}{X}\right) \\
+\frac{1}{\pi(1-v) \eta X} P V \int_{0}^{a} q\left(X^{\prime}\right) \sqrt{\frac{a-X^{\prime}}{a-X}} \frac{\mathrm{d} X^{\prime}}{X^{\prime}-X} \\
=-\frac{\left(\mathcal{A}_{23}+2 v \mathcal{A}_{13}\right) K_{I}-\mathcal{A}_{33} K_{I I I}}{2 \sqrt{2 \pi} X^{3 / 2}} .
\end{array}\right.
$$

In both cases, these integro-differential equations on $p(X)$ and $q(X)$ have to be completed by the following boundary condition:

$$
\left\{\begin{array}{l}
p(a)=\frac{K_{I}}{\sqrt{2 \pi a}} \\
q(a)=\frac{K_{I I I}}{\sqrt{2 \pi a}} .
\end{array}\right.
$$

The factors $\mathcal{A}_{p q}$ are linked to the behavior of the $\mathrm{CZ}$, hence to the geometry of the facets in the inner problem, implying that $\mathcal{A}_{p q}=\mathcal{A}_{p q}(\alpha, \ell / d)$. The plots of $\mathcal{A}_{p q}(\alpha)$ for several values of $\ell / d$ deduced from Lazarus et al. (2020) are given in Appendix A. Their definition is also recalled in there. Rewritting Eqs. 3 or 4, and Eqs. 1 and 2 on the following dimensionless quantities $X^{*}=\frac{X}{a}, p^{*}=\frac{p \sqrt{2 \pi a}}{K_{I}}, q^{*}=\frac{q \sqrt{2 \pi a}}{K_{I}}$, it becomes clear that $p^{*}, q^{*}$ and thus $G_{e f f} / G$ are functions of $\alpha, \ell / d, \eta, K_{I I I} / K_{I}, v$.

\subsection{Application to the determination of the fracture threshold}

Suppose that the fracture energy cost per unit surface, $G_{c}$, is independant of the mode mixity, that is the same in pure mode I than in mode I+III. Following Griffith criterion, the crack is able to propagate only if the energy release rate equals $G_{c}$. In a three dimensional setting, this condition has to be fullfilled locally at each point of the crack front line. The surface fracture energy $G_{c}$ is linked to the mode I fracture toughness $K_{c}$ by $G_{c}=\frac{1-v^{2}}{E} K_{c}^{2}$. These quantities are classical material constants which are known for a wide range of materials (Ashby, 1989).

Consider a straight crack front subjected to uniform mixed mode I+III loading, through some remote force $F$ applied somewhere on the boundary of the body. We suppose that propagation occurs in two steps: First, facets appear; Second the facets advances once a peak load $F_{c}$ is reached until the final breakage of the specimen. At this point, we assume (i) that the advance of all the points along the facets is ruled by Griffith threshold $G_{c}$; (ii) that no ligaments between the facets has formed that would induce an additional energy penalty (Lin et al., 2010). We aim here to compare $F_{c}$ with the threshold $F_{0}$ required for uniform coplanar propagation.

Determination of $F_{0}$ Denote $K_{I}^{(1)}, K_{I I I}^{(1)}$ the SIF and $G^{(1)}$ the energy release rate along the initial crack for a unit force $F=1$. Then, using the linearity of $K_{I}$ and $K_{I I I}$ with $F$, together with Irwin's formula (Eq. 2), the loading $F_{0}$ required to trigger coplanar propagation verifies $F_{0}^{2} G^{(1)}=G_{c}=\frac{1-v^{2}}{E} K_{c}^{2}$ that is

$$
F_{0}^{2}=\frac{G_{c}}{G^{(1)}}=\frac{K_{c}^{2}}{\left(K_{I}^{(1)}\right)^{2}+\frac{1}{1-\nu}\left(K_{I I I}^{(1)}\right)^{2}}
$$

Determination of $F_{c}$ Once facets appeared, the propagation of the crack in the direction $X$ corresponds to the advance of the tips of the facets in this direction. In the external problem of the $\mathrm{CZ}$ model, these tips correspond to the 
tip of the $\mathrm{CZ}$ and $G_{e f f}$ to their effective energy release rate. Taking into account that the effectively broken surface is $\ell$ over one period $d$, we obtain $F_{c}^{2} G_{e f f}^{(1)} d=\ell G_{c}$ where $G_{e f f}^{(1)}$ is the effective fracture energy for $F=1$, so that:

$$
F_{c}^{2}=\frac{\ell}{d} \frac{G_{c}}{G_{e f f}^{(1)}}
$$

From Eqs. (6) and (7), it follows that

$$
\frac{F_{c}}{F_{0}}=\sqrt{\frac{\ell}{d} \frac{G^{(1)}}{G_{e f f}^{(1)}}}=\sqrt{\frac{\ell}{d} \frac{G}{G_{e f f}}},
$$

the last equality being justified by the independence of $\frac{G}{G_{e f f}}$ with the applied force.

We have observed in Lazarus et al. (2020) that even if $p$ and $q$ depends on $v$, its influence on $G_{e f f}$ is weak. The ratio $F_{c} / F_{0}$ can thus in practice be determined from the knowledge of the facet geometry $(\alpha, d / a, \ell / d)$ and the mixity factor $K_{I I I} / K_{I}$, by

1. determining the solution $p, q$ of Eqs. (3) or (4) completed by the boundary conditions Eq. (5);

2. inserting this solution into Eqs. (1), Irwin's formula (Eq. 2) and finally Eq. (8).

Eq. (8) highlights that two terms contribute to the difference between $F_{c}$ and $F_{0}$ : the ratio of broken surface $\ell / d$ and $G / G_{e f f}$. While $G / G_{e f f}$ is related to the solution of the elasticity problem depicted in Fig. $2, \ell / d$ is a contribution capturing the difference in fracture costs correlated to the broken surface area. Since $G / G_{e f f}$ is always greater than 1 due to the pinning forces induced by the ligaments between the facets (Leblond et al., 2015; Lazarus et al., 2020), it always participates to an increase of the apparent toughness. In contrary, the first contribution $\ell / d$ induces an increase only if $\ell / d \geq 1$, that is the surface broken by the facet is larger than a coplanar propagation would induce.

\section{Experimental evidence of toughening induced by the facets}

\subsection{4 point bending experiments with an inclined notch}

As in some of our previous studies (Lazarus et al., 2008; Chen et al., 2015; Cambonie et al., 2019), experiments are carried out using cast ${ }^{1} \mathrm{PMMA}^{2}$ beams and a traditional four point bending setup (Fig. 3). The beams of length $L=50 \mathrm{~mm}$ have a squared cross-section of width $W=10 \mathrm{~mm}$. An initial slit is manufactured by micro-milling (diameter $400 \mu \mathrm{m}$ ) and further sharpened by pushing a razor blade inside the gap controlling the applied load by a mechanical testing machine. The length of the added sharp notch is approximately $1 / 10$ of the total length $a_{0}$ of the crack. The residual stresses introduced by this process, are relaxed by heating. More details about the manufacturing of the samples are given in Appendix B. To introduce some amount of mode III, the crack is tilted with an angle $\Gamma_{0}$ from the mode I central plane of symmetry (that corresponds to $\Gamma_{0}=0$ ). When $\Gamma_{0}$ increases, the amount of shear mode III increases.

The sample is centered thanks to a 3D printed support, in an Instron 4 point bending device (Instron 5882 mounted with $1 \mathrm{kN}$ force sensor). The distance between the upper loading points is fixed to $L_{1}=40 \mathrm{~mm}$ and between the lower supporting points $L_{2}=20 \mathrm{~mm}$. An increasing displacement at a loading velocity $V=0.1 \mathrm{~mm} \cdot \mathrm{s}^{-1}$ or $V=0.2 \mathrm{~mm} . \mathrm{s}^{-1}$ is applied (without visible impact of $V$ on our results) until the sample breaks abruptly into two pieces at a critical force $F_{c}$ (see Fig. 3 for a typical loading curve). We perform experiments for different values of $a_{0}=2,3,4 \mathrm{~mm}$ and tilting angle $\Gamma_{0}$ ranging from 0 to $30^{\circ}$. For each experiment, $F_{c}$ is measured as the maximum load. The evolution of $F_{c}$ with $\Gamma_{0}$ for different values of $a_{0}$ is given in Fig. 4 . One notices an increase of $F_{c}$ with $\Gamma_{0}$ and a decrease with $a_{0}$. The different symbols used for the points are linked to different types of fracture surface morphologies that will be defined below, in Section 2.3.

\footnotetext{
${ }^{1}$ obtained by casting the polymer. This fabrication process guaranties that the material is isotropic, in contrary to PMMA obtained by extrusion which is in general anisotropic.

${ }^{2}$ Two brands of PMMA Vacour or Abaqueplast have been used with no noticeable difference in the results.
} 


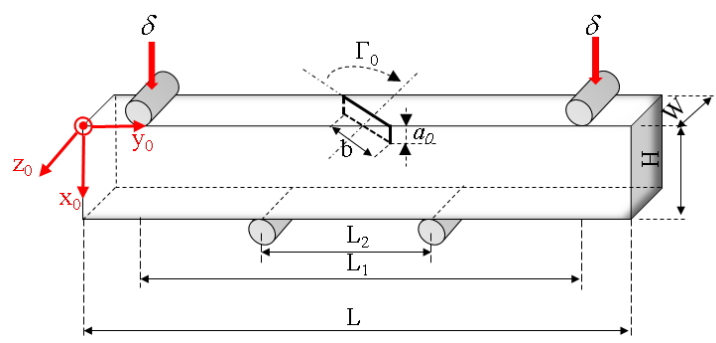

(a) 3D sketch of the setup

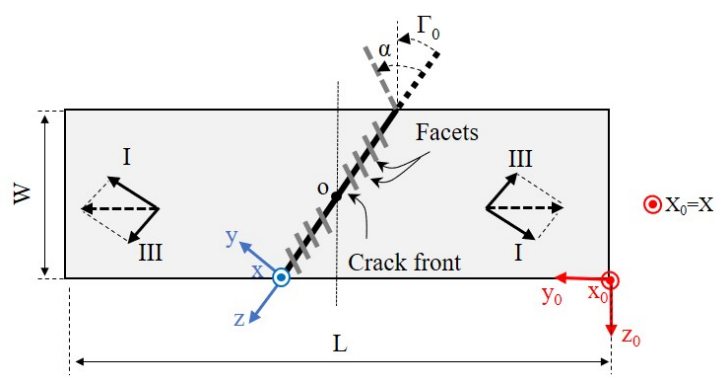

(b) $2 \mathrm{D}$ sketch of the setup and the facets bottom view

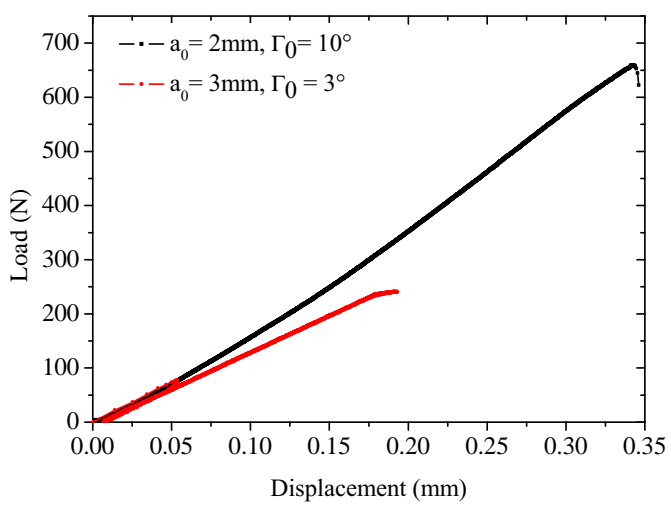

(c) Typical loading curve

Figure 3: (a-b) Geometry and nomenclature of the loading conditions for the notched four-point bending specimen. The $\left(X_{0}, Y_{0}, Z_{0}\right)$ directions are linked to the sample orientation. A local axis system $\left(X=X_{0}, Y, Z\right)$ is associated with the crack front that is tilted with an angle $\Gamma_{0}$ with respect to $X_{0}$. When the crack front is aligned with the pure mode I direction, $\Gamma_{0}=0$. When $\Gamma_{0} \neq 0$, some mode III contribution appears and induces the formation of facets tilted by an angle $\alpha$ toward the shear free direction. Axes and angle notations are chosen so that $\alpha>0, K_{I I I}>0$ when $\Gamma_{0}>0$. (c) Typical experimental loading curves with a linear increase of the force with the applied displacement until the final breakage of the sample. 


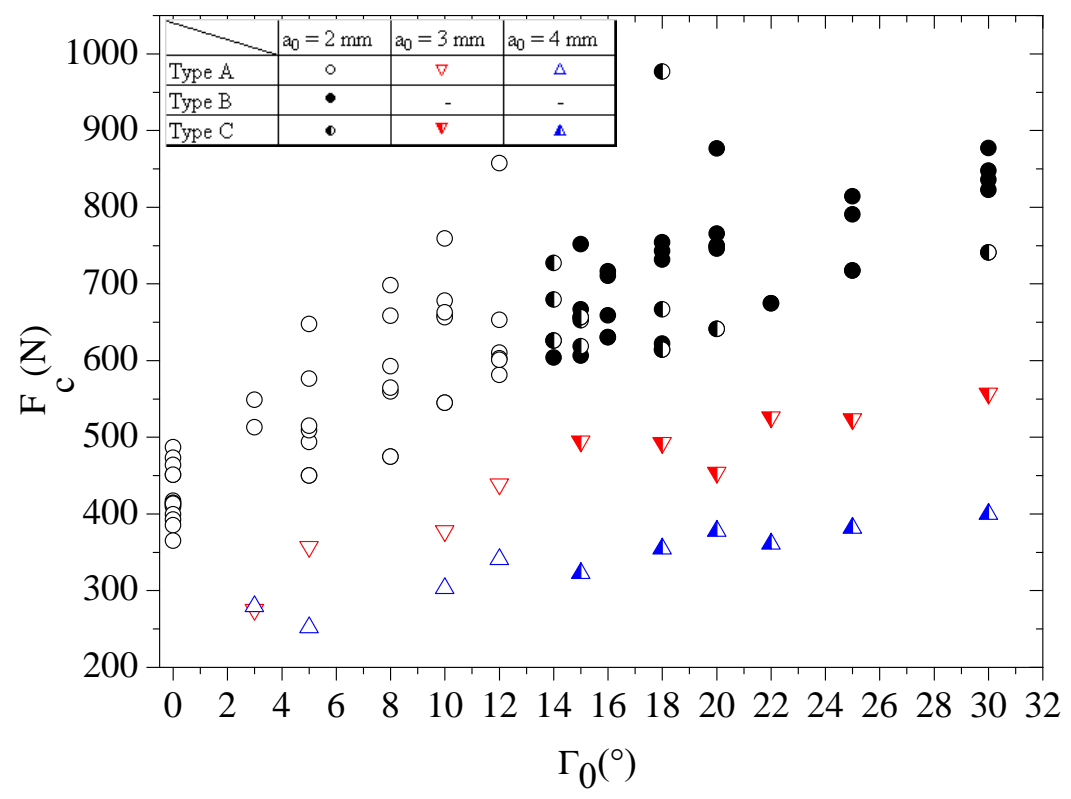

Figure 4: Maximum load $F_{c}$ measured for several values of $\Gamma_{0}$ and $a_{0}$.

\subsection{Finite Element calculation of the Stress Intensity Factors}

The evolution of the Stress Intensity Factors $(\mathrm{SIF}) K_{I}(Z), K_{I I}(Z), K_{I I I}(Z)$ along the crack front are computed by Finite Elements in the frame $(0, X, Y, Z)$ defined in Fig. $3 \mathrm{~b}$, for different values of $\Gamma_{0}$ and $a_{0}$. Software Abaqus relying on interaction integrals (Shih and Asaro, 1988) is used. These functions $K_{I}(Z), K_{I I}(Z), K_{I I I}(Z)$ are given in Appendix $\mathrm{C}$ for some selection of $a_{0}$ and $\Gamma_{0}$. For symmetry reasons, $K_{I I}$ is an odd function of $Z$, hence $K_{I I}(Z=0)=0$ (the origin 0 of the frame is defined in Fig. 3). Also in a wide zone in the middle of the front, $K_{I I I} / K_{I}$ is almost constant and $K_{I I} / K_{I I I}$ remains small. In the sequel, we choose to quantify the mode mixity $K_{I I I} / K_{I}$ by the value $K_{I I I} / K_{I}(Z=0)$. We get a remarkable fit with the FE values using:

$$
\frac{K_{I I I}}{K_{I}} \equiv \frac{K_{I I I}}{K_{I}}(Z=0) \simeq \kappa a_{0}^{-0.4} \tan \Gamma_{0}
$$

with $\kappa=0.95 \mathrm{~mm}^{0.4}$

\subsection{Fracture patterns}

In these experiments the fracture occurs abruptly once the threshold is reached, so that in-situ observation of the propagation path is impossible contrary to the experiments of Fig. 1 that were performed on similar specimens (same material and geometry) but under cyclic loading (Chen et al., 2015). Looking at the broken samples, three types of fracture patterns can be observed (Fig. 5) corresponding either to:

Type A The formation of a factory roof surface along the whole crack front with river lines that coalesce during propagation. These features are similar to the ones observed during fatigue crack propagation (Fig. 1c) and are the traces left by the formation of the facets and the further rupture of the ligaments between them (Lin et al., 2010). These facets are pictured by the case $\Gamma=8^{\circ}$ on the left of Fig. 5 .

Type B The failure of the sample into two pieces by the propagation of a new crack initiating from one single point randomly located along the crack front. No facets apparition along the initial front is observed. This feature is illustrated by the case $\Gamma=30^{\circ}$ on the right of Fig. 5 . 


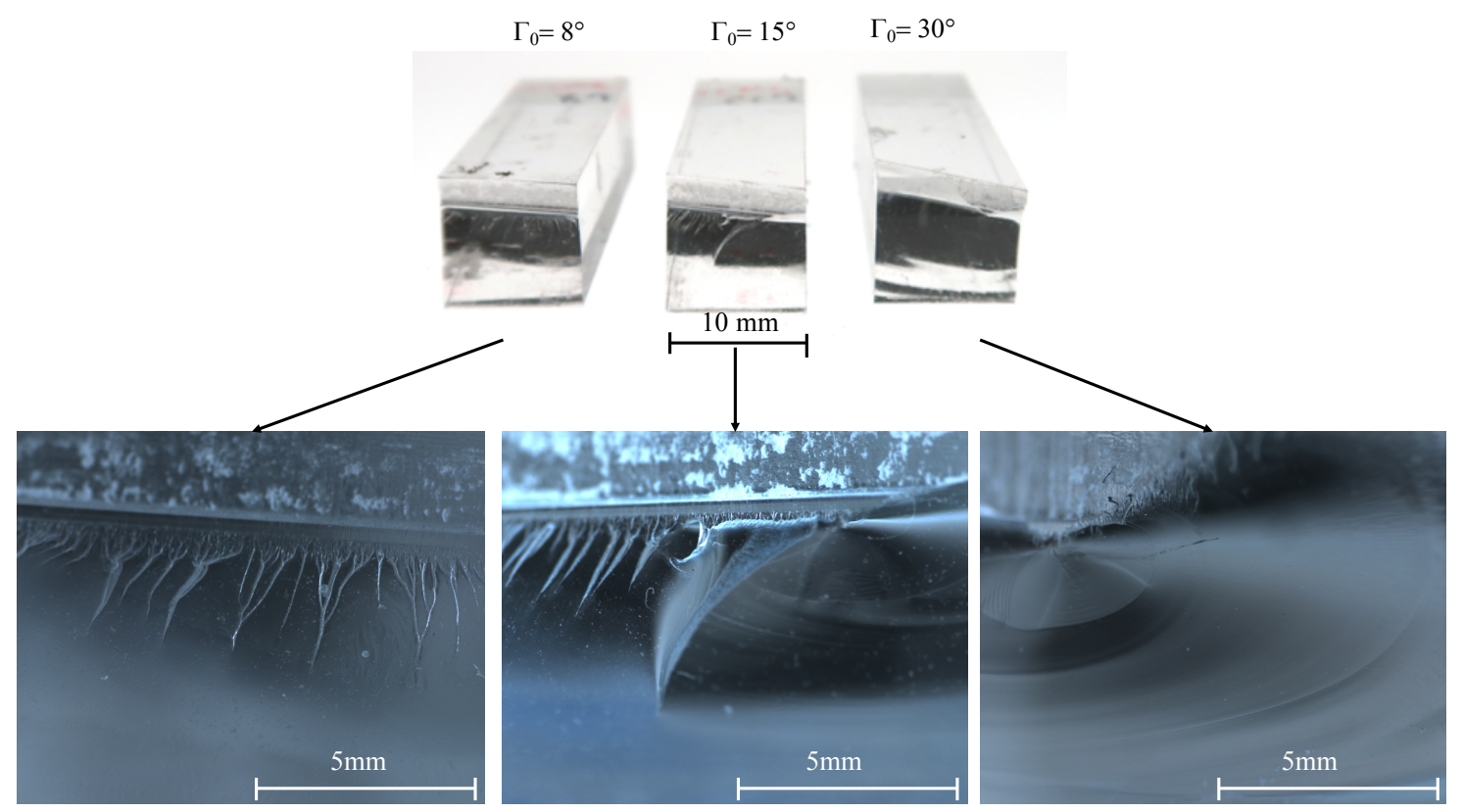

Figure 5: Fracture surfaces as observed by numerical microscopy. $\Gamma_{0}=8^{\circ}$ corresponds to Type A, for $\Gamma_{0}=15^{\circ}$ to Type $\mathrm{C}$, for $\Gamma_{0}=30^{\circ}$ to Type B.

Type C A blend of the two precedent patterns: factory roof patterns along some part of the front, while the other part is broken by a plane which is not in continuity with the initial crack front, as can be observed on the case $\Gamma=15^{\circ}$ in the middle of Fig. 5.

In Fig. 4, the symbols refer to these different types. One can notice that:

- below a value $\Gamma_{c} \simeq 13^{\circ}-15^{\circ}$, all samples are of type $\mathrm{A}$.

- for larger values of $\Gamma_{0}$, different types coexist for the same value of $a_{0}$ and $\Gamma_{0}$ (see for instance $a_{0}=2 \mathrm{~mm}$ and $\left.\Gamma_{0}=15^{\circ}\right)$.

We have also done some preliminary experiments for other values of $W$, namely $W=12,15,20 \mathrm{~mm}$, keeping the aspect ratio of the samples, and noticed no significative effect on $\Gamma_{c}$.

\subsection{Quantification of the facet geometry}

Focusing on type A $\left(\Gamma_{0}<\Gamma_{c}\right)$, the facet rotation angle $\alpha$ and the facet aspect ratio $\eta$ have been obtained by profilometry. The experimental setup and measurement protocol, as well as the data's post-processings, are thoroughly detailed in Cambonie and Lazarus (2014). We only provide here a summary for brevity's sake. The fracture profiles have been scanned every $25 \mu \mathrm{m}$ from the tip of the precrack, which is set as the origin along the propagation direction $(a=0 \mathrm{~mm})$. Notice that this position presents some uncertainty since the roughness of the initial slit and facets are difficult to distinguish as long as their sizes are comparable. Fig. 6 is a summary of the procedure used corresponding to the particular, but representative, case $\Gamma_{0}=12^{\circ}$. Fig. 6a displays an example of 2D fracture profil taken at the position $a=0.61 \mathrm{~mm}$. The extrema are detected (the orange markers in Fig. 6a) and used to define each facet. The mostly sharp profile of the facets makes it possible to approximate each facet by the line between pairs of minimummaximum (the dotted orange lines in fig. 6a). From it, we retrieve each facet's tilt angle $\alpha_{i}$, and the distance between consecutive facets $d_{i}$. Therefore for each position $a$ along the $x$-axis, distributions of tilt angles $\left\{\alpha_{i}\right\}_{(x=a)}$ and distances $\left\{d_{i}\right\}_{(x=a)}$ are obtained, and are represented using black x markers in Fig. 6b, c. In these figures, the mean value at each position is computed and is shown using the red line to emphasize the evolution of the facet characteristics with the distance of propagation. 


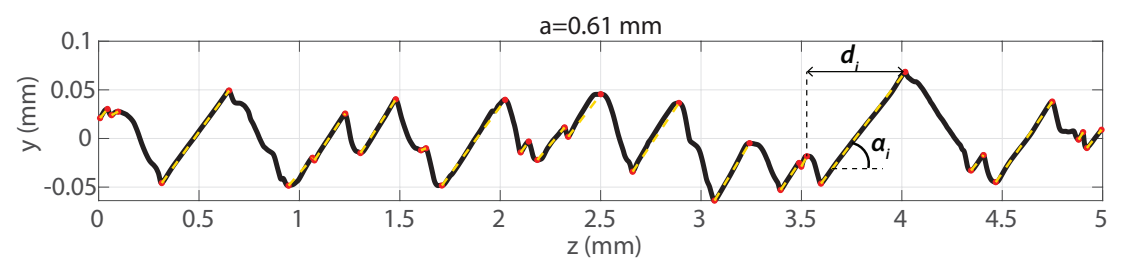

(a)

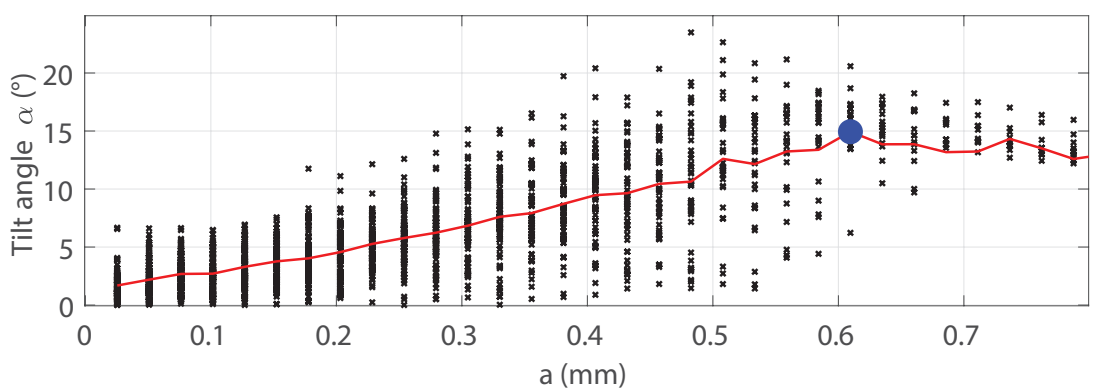

(b)

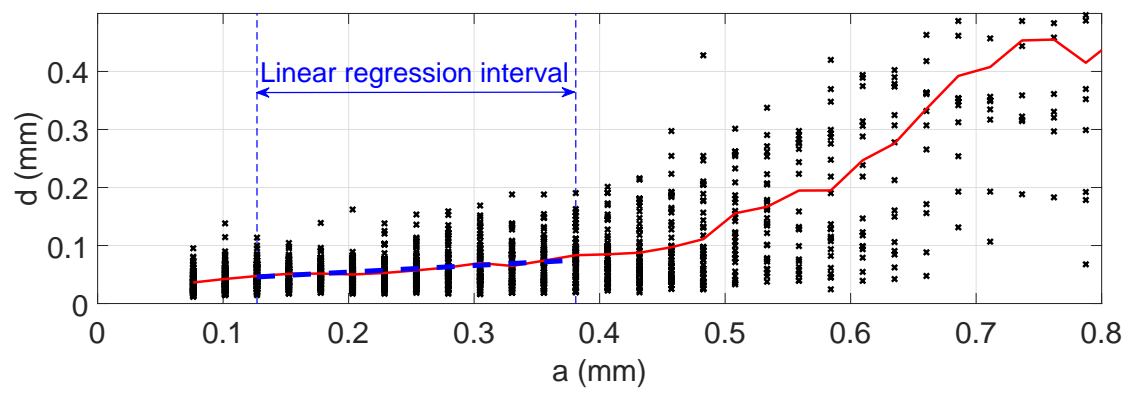

(c)

Figure 6: Outline of the procedure to get the tilt angle $\alpha$ and the coarsening rate $\eta$ from the fracture surface measured using a profilometer (in this example $\Gamma_{0}=12^{\circ}$ ): (a) Profile of the crack surface along a line located at a distance $a$ of the initial slit. The facets are highlighted in orange. (b, c) Evolution of the tilt angle and distance between two facets. Each black marker corresponds to one single facet at a given distance $a$. The red line is their mean value. The maximum value of $\alpha$ is marked by a blue dot. The range that is considered for linear interpolation of $d(a)$ is located before the sharper increase and the best fit of the red curve is plotted in blue dashed line. 
The procedure to characterize the tilt angle and coarsening rate of one experiment by a unique representative and objective value is defined as follows. In all the experiments, the tilt angle is observed to increase continuously until a maximum value (Marker: blue circle in Fig. 6b). This maximum is reported on Fig. 7a as a function of $K_{I I I} / K_{I}$ using Eq. 9. The distance $d$ between the facets always presents a linear increase with $a$ before a sharper increase (Blue line in Fig. 6c). We determine its slot by linear regression and report its value as $\eta\left(K_{I I I} / K_{I}\right)$ in Fig. 7c.

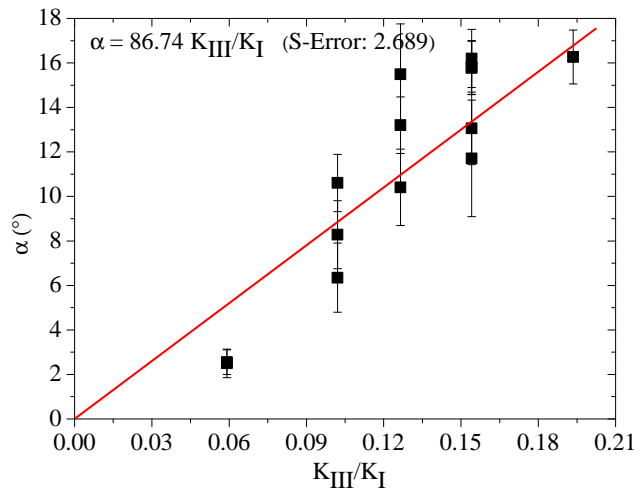

(a)

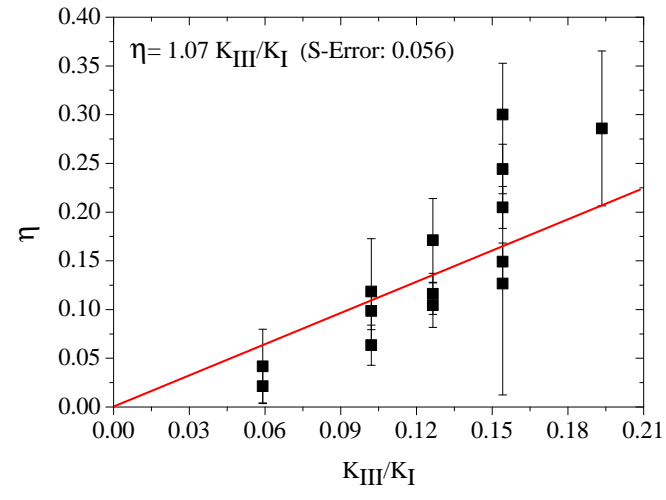

(b)

Figure 7: (a) The tilt angle $\alpha$ is taken as the maximum value of $\alpha(a)$ (blue dot in Fig.. 6b), the error bars corresponds to the standard deviation of the facet angle distribution at the peak location. (b) The coarsening rate $\eta$ is taken as the slop of the linear part of the evolution $d(a)$ (in blue in Fig.. 6c), the error bars corresponds to the upper and lower bounds of the $95 \%$ confidence interval of the linear regression.

Interpolation of these points, using that $\alpha=0$ and $\eta=0$ when $K_{I I I} / K_{I}=0$, gives the following linear approximation:

$$
\begin{aligned}
& \alpha=86.7 \frac{K_{I I I}}{K_{I}} \text { degrees } \\
& \eta=1.07 \frac{K_{I I I}}{K_{I}}
\end{aligned}
$$

The complete determination of the facet geometry requires moreover the knowledge of $\ell / d$. But, unfortunately it is not possible to get it for our samples for two reasons: (i) In-situ measurement during propagation is impossible due to the unstable propagation of the crack; (ii) The ultimate propagation of the ligaments between the facets erases the initial width $\ell$ of the facets, so that it cannot be measured postmortem contrary to $\alpha$ and $\eta$.

\section{Application of the Cohesive Zone model to the experiments}

Fig. 8 aims to compare experiments with the $\mathrm{CZ}$ model using the values of $F_{c} / F_{0}$ as function of the mode mixte ratio $K_{I I I} / K_{I}$. The building of this figure is quite complexe. It follows the method described below:

- From a theoretical point of view, plugging the experimental values (Eqs. 10) of $\alpha$ and $\eta$ into Eq. 8 gives $F_{c} / F_{0}$ as a function of $K_{I I I} / K_{I}$ for given values of $\ell / d$. As explained in Sec. 1.3, it requires to solve Eq. 4 or Eq. 3 to get $p$ and $q$ and then $G_{e f f} / G$. The values are different whether assuming coalescence (that is solving Eq. 4) or not (solving Eq. 3). The functions $\mathcal{A}_{p q}$ have not been calculated yet neither for large values of $\ell / d$, nor for small values of $\alpha$ if $\ell / d \sim 1$, since proximity of the tips would require much higher level of refinement in the calculations (Lazarus et al., 2020). This explains why the theoretical curves start at $K_{I I I} / K_{I} \sim 0.05$ (corresponding to $\alpha \sim 5^{\circ}$ ) and no results are provided for $\ell / d>1.3$.

- From an experimental point of view, the critical force $F_{c}$ is given as function of $\Gamma_{0}$ and $a_{0}$ in Fig. 4 . It can be changed to get $F_{c}$ as a function of $K_{I I I} / K_{I}$ using their values obtained by FE simulations (Eq. 9). The load to promote straight propagation $F_{0}$ can not be measured directly since facets always appear. It can be obtained by 


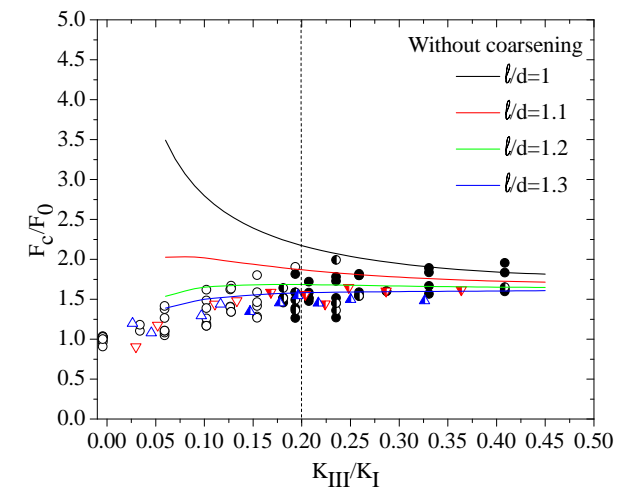

(a) $\mathrm{CZ}$ model without coalescence

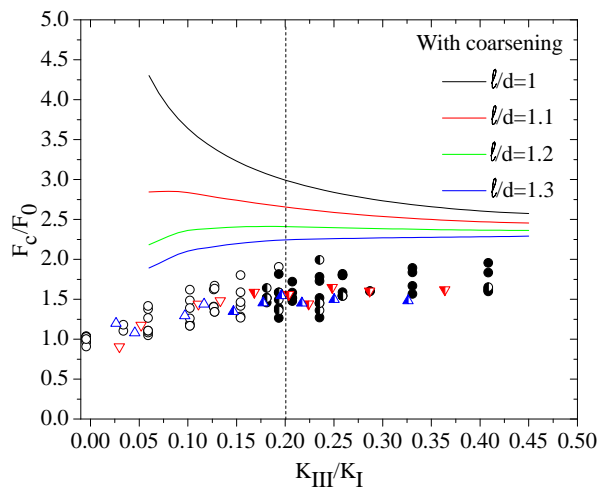

(b) $\mathrm{CZ}$ model with coalescence

Figure 8: Comparison between the $\mathrm{CZ}$ model and the experimental values of $F_{c} / F_{0}$. The point symbols are the same than on Fig. 4 . The $\mathrm{CZ}$ model is applicable only for type A fracture surfaces (empty symbols) that is on the left of vertical line located around $K_{I I I} / K_{I} \sim 0.2$.

Eq. 6 in which we plug (i) the toughness $K_{c}$ obtained by performing mode I experiments $\left(\Gamma_{0}=0\right)$ for several values of $a_{0}$ that is $K_{c}=1.23 \mathrm{MPa}^{0.5}$ (Abaqueplast brand) or $K_{c}=1.06 \mathrm{MPa}^{0.5}$ (Vacour brand); (ii) $K_{I}^{(1)}$ and $K_{I I I}^{(1)}$ computed by Finite Element taking $F=1$ (Appendix C), $v=0.38$ and the geometry of the setup described in Section 2.1. Notice that these SIFs are independent of $E$ for dimensional reasons and thanks to their linearity with $F$.

The CZ model is applicable only if facets appear along the whole crack front, that is only for type A fracture surfaces. Those experiments corresponds to empty symbols in Fig. 8 and are located on the left of the vertical line $K_{I I I} / K_{I}=0.2$. Several observations can be made:

- plotting $F_{c} / F_{0}$ as a function of $K_{I I I} / K_{I}$ instead of $\Gamma_{0}$ and $a_{0}$ as in Fig. 4, induces a collapse of the experimental points. This shows that $K_{I I I} / K_{I}$ is the pertinent parameter to estimate the local load along the initial slit.

- A relatively large spread in this collapse, that can be attributed to the variability of the measure of the peak force. This measure is very sensitive to any imperfection, notably of the initial slit, even though we took great care in its manufacturing (see Appendix B). This variability has still be observed in PMMA with a slit prepared in a similar way (Maccagno and Knott, 1989) and may be amplified here in presence of mode III by the nucleation of new cracks (the facets), that is known to be sensitive to the randomness of defects.

- While the experiments show an increase of the apparent toughness with $K_{I I I} / K_{I}$, this behavior is caught by the $\mathrm{CZ}$ model only if $\ell / d \geq 1.2$, meaning that the interaction between adjacent cracks plays an important role.

- The model with coalescence overestimates the experimental values, while a very good agreement is found for the model without coalescence. This result sounds surprising at first glance because coalescence is visible on the postmortem face, but is consistent with the fact that $F_{c}$ is measured experimentally at the very beginning of facet formation, whereas coalescence has probably not yet had time to express itself.

- The aspect ratio of the facets $\ell / d$ has to be adjusted to $\ell / d=1.2-1.3$ to be in agreement with the experiments. This is reasonable in view of the values that can be measured in-situ on fatigue experiments (Fig. 1b).

The good agreement between the $\mathrm{CZ}$ model and experimental values of $F_{c} / F_{0}$ shows that the apparent toughening observed in Fig. 4 can be attributed mainly to the presence of the facets at the microscale and can be determined from the knowledge of their geometry via the $\mathrm{CZ}$ model. The sole material constants required to get $F_{c}$ are the standard ones, namely Poisson's ratio $v$ and the mode I toughness $K_{c}$ (or equivalently the fracture surface energy $G_{c}$ and Young modulus $E$ ). It was useless here to resort to any additional constant, as for instance a specific mode III toughness $K_{I I I c}$ 
for mode III propagation or similarly a dependance of $G_{c}$ with $K_{I I I} / K_{I}$ (Lin et al., 2010; Leblond et al., 2019). In other words, it means that in our experiments, the apparent dependence of $G_{c}$ with $K_{I I I} / K_{I}$ comes from the dependence of the facet geometry with $K_{I I I} / K_{I}$.

\section{Discussion}

\subsection{Determination of the facet geometry}

In this study, we have seen that the $\mathrm{CZ}$ method permits to quantify the toughening effect knowing the facet geometry through the constants $\alpha, \ell / d$ and $\eta$. This reports the problem on the determination of these constants. Selfsustainibility of the method requires that they can be obtained from the knowledge of the load and $K_{c}, E, v$. In Lazarus et al. (2020), we proposed:

- To determine the tilt angle $\alpha$ using either the Principle of Local Symmetry or energy minimisation; both have been observed to yield very similar values and to be in agreement with some experiments, but more extensive comparison remains to be done notably by monitoring carefully the value of the Poisson' ratio $v$;

- That the lateral extension $\ell / d$ results from the application of Griffith minimisation principle. Comparison with experiments necessitate in-situ measurements of $\ell / d$. It can only be done if the crack advances slowly, that was not the case here.

Hence $\alpha$ and $\ell / d$ can be anticipated to depend only on $K_{c}$ and the elastic constants $E, v$, so that the fully efficiency of the method, relies on the determination of $\eta$. Its is the most challenging since it necessitates to solve a three dimensional problem to get the 3D shape of the facets. Whether it does or not necessitate an additional material constant remains an issue (Chen et al., 2015). In any case, careful and extensive comparisons with dedicated experiments has to be performed to completely validate any of these propositions (Lazarus et al., 2020).

In all this study, we assume that the facets are straight, but it is likely (Ghelichi and Kamrin (2015); Chen et al. (2015), supplemental material) that en-passant curved shapes appear as in the experiments of Fig. 1. Phase-field simulations of the internal problem may be used to get this shape (Mesgarnejad et al., 2019) and at the same time the corresponding $\mathrm{CZ}$ model, that is the functions $\mathcal{A}_{p q}$ involved in Eqs. 3 and 4. This task can be anticipated to be highly demanding since the outcome of the CZ model is highly sensitive to $\ell / d$ for small values of $\alpha$. This is because the adjacent crack tips in the internal problem (Fig. 2) are then very close hence very sensitive to their precise location in particular in case of overlapping $(\ell / d \sim 1)$. This high sensitivity also explains the high variability of $F_{c} / F_{0}$ with $\ell / d$ that can be observed in Fig. 8 for small values of $K_{I I I} / K_{I}$, that corresponds to small values of $\alpha$ (Eq. 10). This point is discussed further in Appendix A.

\subsection{Predominant toughening mechanism}

Section 1.3 and Eq. (8) evidence that the facets contribute to the toughening through two physical mechanisms: (i) changes in fractured surface area $\ell / d$ in comparison to a straight propagation, and (ii) crack shielding, that is the effective decrease of the load $G_{e f f} / G$ in the vicinity of the facets due to partial breaking of the cohesive zone. We aim here to discuss their relative importance.

For this, consider the theoretical evolution of $F_{c} / F_{0}$ with $\ell / d$, keeping $K_{I I I} / K_{I}$ constant (that is $\alpha$ and $\eta=d / a$ as well). One observes on Fig. 8 that $F_{c} / F_{0}$ diminishes with increasing $\ell / d$, in other words that the correlation between $F_{c} / F_{0}$ and $\ell / d$ is negative. Remembering that $F_{c} / F_{0}$ is computed from Eq. (8) involving positive correlation with $\ell / d$ and $G / G_{e f f}$. It means that this negative correlation can only be attributed to a negative correlation between $G / G_{e f f}$ and $\ell / d$, that is to the increase of $G_{e f f} / G$ with $\ell / d$. Looking at Fig. 2 by fixing $d, a$ (that is $\eta$ ) and $\alpha$, this increase can itself easily be understand by the increase of the opening of the Cohesive Zone when the width of the facets $\ell$ increases. While it is clear that the increase of fracture surface area contributes to the increase of the apparent toughness (Kolvin et al., 2017) since it induces an increase of the fracture costs, the necessity to involve $G_{\text {eff }} / G$ to explain the behavior of $F_{c} / F_{0}$ with $\ell / d$, shows that besides the fracture costs, $G_{e f f} / G$ that is, the modification of the apparent energy release rate by crack shielding cannot be dismissed and even plays a determinant role. The CZ model is an efficient way to quantify this decrease without resorting to costly simulations of the 3D elasticity problem in the presence of facets. Conciliating the microscopic scale of the facets with the macroscopic structure can even be anticipated to be unfeasible in FE simulations without using multi-scale approaches. 


\section{Conclusion and perspectives}

In the continuity with our previous experimental campaigns (Lazarus et al., 2008; Leblond and Lazarus, 2015; Chen et al., 2015; Lazarus et al., 2020), we have conducted bending experiments on PMMA beams containing an inclined initial slit, inducing some mode III shear load along the crack front. Here, instead of applying a cyclic loading to study fatigue propagation, the specimens were loaded until the abrupt rupture of the specimen into two pieces. We measured the critical load for various orientation and depth of this slit. We observed (i) some river-line patterns on the post-mortem crack surface, that are analog to those observed in fatigue and that can be attributed to the formation and further coalescence of facets/segments during propagation; (ii) an increase of the critical load with the inclination of the slit that is, with increasing $K_{I I I} / K_{I}$ ratio.

Comparing the results with a two-scale Cohesive Zone (CZ) model that we have developed recently (Leblond et al., 2015; Lazarus et al., 2020), we showed that

- this apparent toughening can be attributed to the presence of facets at the microscale, whose further propagation require a higher load than the one that promotes a smooth propagation.

- resorting to an additional mode III material constant is useless; the apparent dependence of the toughness with $K_{I I I} / K_{I}$ can be rationalized through the dependence of the facet geometry with $K_{I I I} / K_{I}$.

- two related physical mechanisms are generated due to the formation of a disconnected crack front: (i) changes in fractured surface area in comparison to a straight propagation, and (ii) crack shielding, that is the decrease of the load in the vicinity of the facets due to reduced opening of the CZ. We show that the second plays an essential role, but is more complex to take into account since it relies on the 3D elasticity problem involving the microscale of the facets and the macroscale of the body. The two-scale CZ model (Leblond et al., 2015; Lazarus et al., 2020) has been demonstrated to be an efficient analytical way to catch the second mechanism.

- the critical load can be determined accurately by the CZ model assuming the coalescence had no time to occur. In practice, from the knowledge of the facets geometry, that is the rotation angle $\alpha$ of the facets and their aspect ratios, $\ell / d$ and $\eta=d / a, p$ and $q$ can be computed solving Eqs. (3) and (5), and then using Eqs. (1), (2) and (8), to get $K_{I}^{e f f}, K_{I I I}^{e f f}, G_{e f f}$ and finally $F_{c} / F_{0}$.

In the present study, the facets geometry was measured using profilometry on the post-mortem fracture surfaces following the procedure described in Cambonie and Lazarus (2014). But, to obtain self-sufficient prediction of the critical loading, the determination of the facets geometry from the loading conditions is necessary. Avenues for determining $\alpha$ are discussed in Pham and Ravi-Chandar (2014); Lazarus et al. (2020), but the determination of $\eta$ and $\ell / d$ will no doubt require to predict numerically the propagation of facets in all their three-dimensional complexity. We envisage it using a phase-field approach in line with the preliminary results presented in Chen et al. (2015) or Pham and Ravi-Chandar (2017).

Additional experiments are necessary (i) to validate the approach for a larger range of $K_{I I I} / K_{I}$ than in our setup; (ii) to drive the fracture quasi-statically, in order to permit in-situ observations of the development of the facet geometry and notably the measure of $\ell / d$. One option is to use the experimental hydraulic fracture setup of Wu et al. (2007). It presents the advantage to involve an internal circular crack, hence to avoid the apparition of mode II inherent to the coupling between mode II and III at a free surface (Dhondt et al., 2001), but the disadvantage to require to extend CZ model to circular crack geometries. Closer perspective is to apply the CZ model to the same kind of experiments in fatigue. This requires careful monitoring of the crack advance with the number of loading cycles (Ben Hadj Khaled, 2018).

\section{CRediT authorship contribution statement}

M. L. Hattali :Experimental data analysis, Finite Element Simulations, Writing - appendix. T. Cambonie: Conceptualization and realization of the experiments. V. Lazarus: Conceptualization, Funding acquisition, Formal analysis, Writing - main part. 


\section{Acknowledgements}

We thank the FAST technical support, notably Lionel Auffray, Raphäl Pidoux and Alban Aubertin, for their help in the experimental setup, Jean-Baptiste Leblond for fruitful discussions, and Florian Marconi, Martin Poncelet, Bumedijen Raka for some inspiring discussions about the experiments. We also thank the reviewers for their throughfull reading, and notably reviewer $\# 2$ for pertinent suggestions of additional references and help to clarify some terminology.

We acknowledge the support of ANR GeoSmec (ANR-12-BS06-0016).

\section{References}

Andrieu, A., 2013. Mécanismes et modélisation multi-échelle de la rupture fragile trans- et inter-granulaire des aciers pour réacteurs à eau sous pression, en lien avec le vieillissement thermique. Theses. Ecole Nationale Supérieure des Mines de Paris. URL: https://pastel.archives-ouvertes.fr/pastel-00957868.

Ashby, M., 1989. Overview no. 80: On the engineering properties of materials. Acta Metallurgica 37, 1273 - 1293. doi:10.1016/00016160(89)90158-2.

Bazant, Z., Estenssoro, L., 1979. Surface singularity and crack propagation. International Journal of Solids and Structures 15, 405-26.

Ben Hadj Khaled, A., 2018. Etude expérimentale de la vitesse de propagation d'une fissure chargée cycliquement en mode I+III. Master's thesis. Materials \& Engineering Sciences in Paris - MAGIS. Supervisors: L. Hattali, V. Lazarus.

Bonniot, T., Doquet, V., Mai, S., 2018. Mixed mode II and III fatigue crack growth in a rail steel. International Journal of Fatigue doi:0.1016/j.ijfatigue.2018.01.010

Cambonie, T., Klinger, Y., Lazarus, V., 2019. Similarities between mode III crack growth patterns and strike-slip faults. Philosophical Transactions of the Royal Society of London A: Mathematical, Physical and Engineering Sciences 377. doi:10.1098/rsta.2017.0392.

Cambonie, T., Lazarus, V., 2014. Quantification of the crack fragmentation resulting from mode I+III loading. Procedia Materials Science 3, 1816-1821. doi:10.1016/j.mspro.2014.06.293.

Cao, H., Evans, A., 1989. An experimental study of the fracture resistance of bimaterial interfaces. Mechanics of Materials 7, $295-304$. doi:https://doi.org/10.1016/0167-6636(89)90020-3.

Chen, C.H., Cambonie, T., Lazarus, V., Nicoli, M., Pons, A.J., Karma, A., 2015. Crack Front Segmentation and Facet Coarsening in Mixed-Mode Fracture. Physical Review Letters 115, 265503. doi:10.1103/PhysRevLett.115.265503.

Davenport, J.C.W., Smith, D.J., 1993. A study of superimposed fracture modes i, ii and iii on pmma. Fatigue \& Fracture of Engineering Materials \& Structures 16, 1125-1133. doi:https://doi.org/10.1111/j.1460-2695.1993.tb00082.x.

Dhondt, G., Chergui, A., Buchholz, F.G., 2001. Computational fracture analysis of different specimens regarding $3 \mathrm{~d}$ and mode coupling effects. Engineering Fracture Mechanics 68, 383-401. doi:https://doi.org/10.1016/S0013-7944(00)00104-1.

Doitrand, A., Leguillon, D., 2018. Numerical modeling of the nucleation of facets ahead of a primary crack under mode I + III loading. International Journal of Fracture 213, 37-50. doi:10.1007/s10704-018-0305-8.

Eberlein, A., Richard, H., Kullmer, G., 2017. Facet formation at the crack front under combined crack opening and anti-plane shear loading. Engineering Fracture Mechanics 174, 21 - 29. doi:http://doi.org/10.1016/j.engfracmech.2016.12.004. special Issue on Multiaxial Fracture 2016.

Evans, A., Hutchinson, J., 1989. Effects of non-planarity on the mixed mode fracture resistance of bimaterial interfaces. Acta Metallurgica 37, 909-916. doi:https://doi.org/10.1016/0001-6160(89)90017-5.

Frémy, F., 2012. Fissuration par fatigue en mode mixte I+II+III non proportionnel dans l'acier 316L Approche expérimentale et modélisation des effets de la plasticité. Ph.D. thesis. ENS Cachan. URL: https://tel .archives-ouvertes.fr/tel-00826964/file/Fremy2012 .pdf.

Fremy, F., Pommier, S., Poncelet, M., Raka, B., Galenne, E., Courtin, S., Roux, J.L., 2014. Load path effect on fatigue crack propagation in I+II+III mixed mode conditions - part 1: Experimental investigations. International Journal of Fatigue 62, $104-112$. doi:10.1016/j.ijfatigue.2013.06.002. 9th Fatigue Damage of Structural Materials Conference.

Ghelichi, R., Kamrin, K., 2015. Modeling growth paths of interacting crack pairs in elastic media. Soft Matter 11, 7995-8012. doi:10.1039/C5SM01376C.

Goldstein, R., Osipenko, N., 2012. Successive development of the structure of a fracture near the front of a longitudinal shear crack. Doklady Physics 57, 281-284. doi:10.1134/S1028335812070087.

Hull, D., 1995. The effect of mixed-mode I/III on crack evolution in brittle solids. International Journal of Fracture 70, 59-79.

Irwin, G.R., 1958. Fracture. Hand. der Physik. volume IV. Springer, Berlin.

Kolvin, I., Cohen, G., Fineberg, J., 2017. Topological defects govern crack front motion and facet formation on broken surfaces. Nature Materials 17, $140 \mathrm{EP}$-. URL: https://doi .org/10.1038/nmat5008.

Lambert-Perlade, A., Sturel, T., Gourgues, A.F., Besson, J., Pineau, A., 2004. Mechanisms and modeling of cleavage fracture in simulated heat-affected zone microstructures of a high-strength low alloy steel. Metallurgical and Materials Transactions A 35, 1039-1053. URL: https://doi .org/10.1007/s11661-004-0030-y, doi:10.1007/s11661-004-0030-y.

Lazarus, V., 2003. Brittle fracture and fatigue propagation paths of 3D plane cracks under uniform remote tensile loading. International Journal of Fracture 122, 23-46. doi:10.1023/B:FRAC.0000005373.73286.5d.

Lazarus, V., 2011. Perturbation approaches of a planar crack in linear elastic fracture mechanics: a review. Journal of the Mechanics and Physics of Solids 59, 121-144. doi:10.1016/j.jmps.2010.12.006.

Lazarus, V., Buchholz, F.G., Fulland, M., Wiebesiek, J., 2008. Comparison of predictions by mode II or mode III criteria on crack front twisting in three or four point bending experiments. International Journal of Fracture 153, 141-151. doi:10.1007/s10704-008-9307-2. 
Lazarus, V., Leblond, J.B., Mouchrif, S.E., 2001a. Crack front rotation and segmentation in mixed mode I+III or I+II+III - part I: Calculation of Stress Intensity Factor. Journal of the Mechanics and Physics of Solids 49, 1399-1420. doi:10.1016/S0022-5096(01)00007-2.

Lazarus, V., Leblond, J.B., Mouchrif, S.E., 2001b. Crack front rotation and segmentation in mixed mode I+III or I+II+III - part II: Comparison with experiments. Journal of the Mechanics and Physics of Solids 49, 1421-1443. doi:10.1016/S0022-5096(01)00008-4.

Lazarus, V., Prabel, B., Cambonie, T., Leblond, J., 2020. Mode I+III multiscale cohesive zone model with facet coarsening and overlap: Solutions and applications to facet orientation and toughening. Journal of the Mechanics and Physics of Solids 141 , 104007. doi:https://doi.org/10.1016/j.jmps.2020.104007.

Leblond, J., Karma, A., Lazarus, V., 2011. Theoretical analysis of crack front instability in mode I+III. Journal of the Mechanics and Physics of Solids 59, 1872-1887. doi:10.1016/j.jmps.2011.05.011.

Leblond, J.B., Frelat, J., 2014. Development of fracture facets from a crack loaded in mode I+III: solution and application of a model 2D problem. Journal of the Mechanics and Physics of Solids 64, 133-153. doi:10.1016/j.jmps.2013.11.001.

Leblond, J.B., Karma, A., Ponson, L., Vasudevan, A., 2019. Configurational stability of a crack propagating in a material with mode-dependent fracture energy - part I: Mixed-mode I+III. Journal of the Mechanics and Physics of Solids 126, 187 - 203. doi:10.1016/j.jmps.2019.02.007.

Leblond, J.B., Lazarus, V., 2015. On the strong influence of imperfections upon the quick deviation of a mode I+III crack from coplanarity. Journal of Mechanics of Materials and Structures 10, 299-315. doi:10.2140/jomms.2015.10.299.

Leblond, J.B., Lazarus, V., Karma, A., 2015. Multiscale cohesive zone model for propagation of segmented crack fronts in mode I+III fracture. International Journal of Fracture (Special Invited Article Celebrating IJF at 50) 191, 167-189. doi:10.1007/s10704-015-0001-X.

Liechti, K.M., Chai, Y.S., 1992. Asymmetric shielding in interfacial fracture under in-plane shear. Transactions of the ASME. Journal of Applied Mechanics 59, 295-304.

Lin, B., Mear, M., Ravi-Chandar, K., 2010. Criterion for initiation of cracks under mixed-mode I+III loading. International Journal of Fracture 165, 175-188. doi:10.1007/s10704-010-9476-7.

Liu, S., Chao, Y., Zhu, X., 2004. Tensile-shear transition in mixed mode I/III fracture. International Journal of Solids and Structures 41, 6147-6172. doi:10.1016/j.ijsolstr.2004.04.044.

Maccagno, T., Knott, J., 1989. The fracture behaviour of PMMA in mixed modes I and II. Engineering Fracture Mechanics 34 , 65 - 86. doi:10.1016/0013-7944(89)90243-9.

Mesgarnejad, A., Imanian, A., Karma, A., 2019. Phase-field models for fatigue crack growth. Theoretical and Applied Fracture Mechanics 103, 102282. doi:https://doi.org/10.1016/j.tafmec.2019.102282.

Palaniswamy, K., Knauss, W.G., 1975. Crack extension in brittle solids. Mechanics Today, Pergamon Press 4, 87.

Pham, K.H., Ravi-Chandar, K., 2014. Further examination of the criterion for crack initiation under mixed-mode I+III loading. International Journal of Fracture 189, 121-138. doi:10.1007/s10704-014-9966-0.

Pham, K.H., Ravi-Chandar, K., 2016. On the growth of cracks under mixed-mode I + III loading. International Journal of Fracture , 130doi:10.1007/s10704-016-0098-6.

Pham, K.H., Ravi-Chandar, K., 2017. The formation and growth of echelon cracks in brittle materials. International Journal of Fracture 206, 229-244. doi:10.1007/s10704-017-0212-4.

Pollard, D.D., Segall, P., Delaney, P.T., 1982. Formation and interpretation of dilatant echelon cracks. Geological Society of America Bulletin 93, 1291-1303.

Pons, A.J., Karma, A., 2010. Helical crack-front instability in mixed-mode fracture. Nature 464, 85-89. doi:10.1038/nature08862.

Qiao, Y., Argon, A., 2003. Cleavage crack-growth-resistance of grain boundaries in polycrystalline Fe-2\%Si alloy: experiments and modeling. Mechanics of Materials 35, 129-154. doi:https://doi.org/10.1016/S0167-6636(02)00194-1.

Ronsin, O., Caroli, C., Baumberger, T., 2014. Crack front échelon instability in mixed mode fracture of a strongly nonlinear elastic solid. EPL (Europhysics Letters) 105, 34001. doi:10.1209/0295-5075/105/34001.

Shih, C.F., Asaro, R.J., 1988. Elastic-Plastic Analysis of Cracks on Bimaterial Interfaces: Part I-Small Scale Yielding. Journal of Applied Mechanics 55, 299-316. doi:10.1115/1.3173676.

Sommer, E., 1969. Formation of fracture 'lances' in glass. Engineering Fracture Mechanics 1, 539-546.

Vasudevan, A., Ponson, L., Karma, A., Leblond, J.B., 2020. Configurational stability of a crack propagating in a material with mode-dependent fracture energy - part II: Drift of fracture facets in mixed-mode I+II+III. Journal of the Mechanics and Physics of Solids 137 , 103894. doi:10.1016/j.jmps.2020.103894.

Wu, R., Germanovich, L.N., Van Dyke, P.E., Lowell, R.P., 2007. Thermal technique for controlling hydraulic fractures. Journal of Geophysical Research: Solid Earth 112, n/a-n/a. doi:10.1029/2005JB003815. b05209.

Zebar, M.E.M., Hattali, M.L., Mesrati, N., 2020. Interfacial fracture toughness measurement in both steady state and transient regimes using four-point bending test. International Journal of Fracture 222, 123-135. doi:10.1007/s10704-020-00437-3.

\section{Appendix A. Functions $\mathcal{A}_{p q}(\alpha, \ell / d)$ and high sensitivity of the solution to $\ell / d$ for small values of $\alpha$}

The functions $\mathcal{A}_{p q}(\alpha, c / d)$ appearing in Eqs. (3) and (4) are linked to the values of the SIF at the lateral crack tips of the internal problem (Fig. 2b) through some functions $\mathcal{F}_{\lambda}^{p}\left(\frac{\ell}{d}, \alpha\right)$ (Leblond et al., 2015; Lazarus et al., 2020). For small enough values of $\ell / d$, their values have been obtained by Leblond and Frelat (2014) and for larger values by Lazarus et al. (2020). By plugging these results in Eq. (7) of Lazarus et al. (2020), $\mathcal{A}_{p q}(\alpha)$ for several values of $\ell / d$ can be obtained. Their plot is given in Fig. A.9.

Fig. 8 of the main part of the paper highlights the high sensitivity of the effective toughness $F_{c} / F_{0}$ with $\ell / d$, for $\ell / d \geq 1$ and $K_{I I I} / K_{I} \ll 1$. In this range of parameters, we have $\alpha \ll 1$ and a high sensitivity of the functions $\mathcal{A}_{p q}$ with $\ell / d$ is observed in Fig. A.9. This is not surprising since adjacent crack tips are then very close to each others (Fig. 2) 

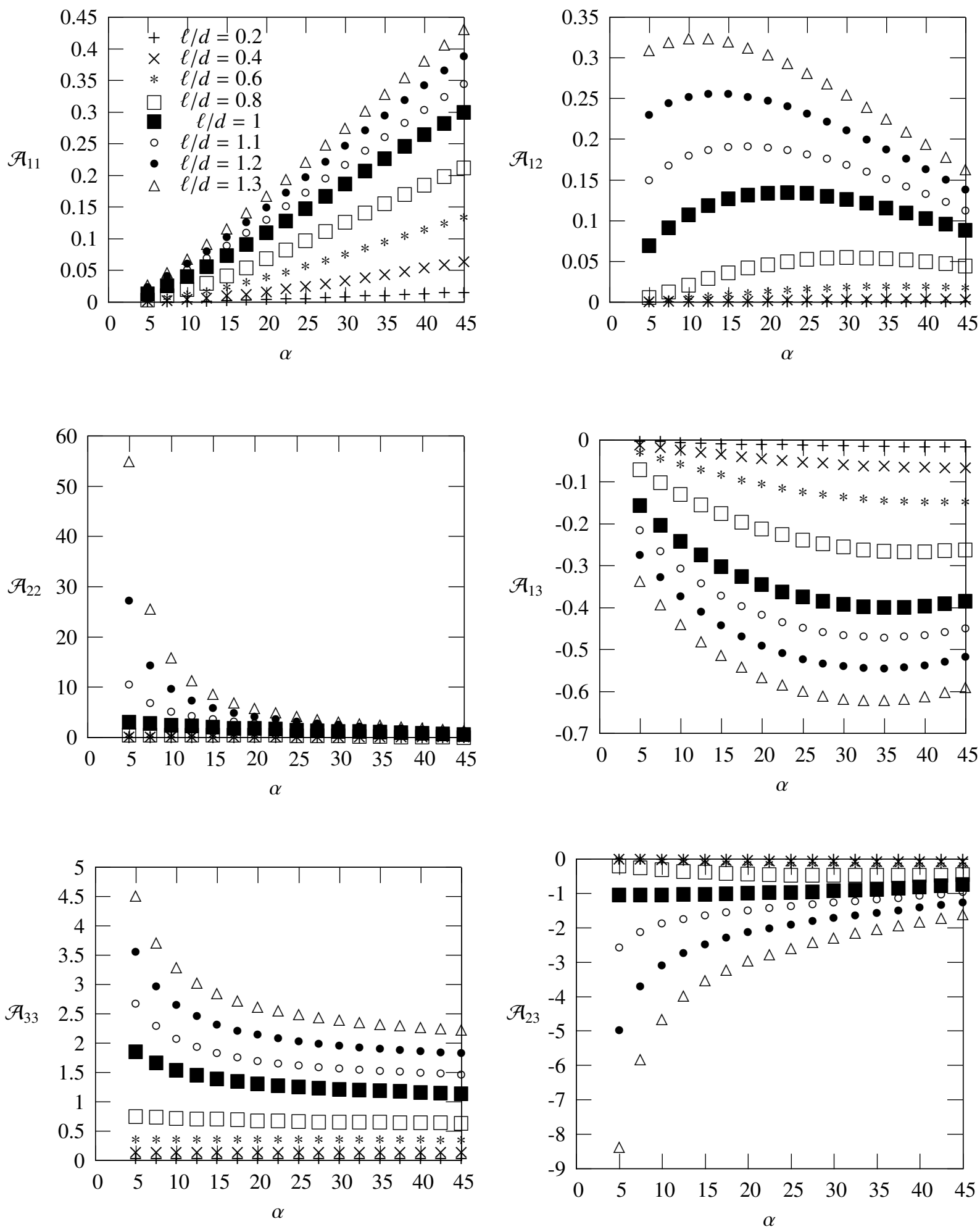

Figure A.9: $\mathcal{A}_{p q}(\alpha)$ for several values of $\ell / d$ 
and their mutual influence becomes very sensitive to any small perturbation in the length $\ell$ of the facets. By the way, for $\alpha<5^{\circ}$ and $\ell / d>1$, the values of these functions have not been computed since sufficient accuracy is then difficult to achieve with finite elements methods (Lazarus et al., 2020).

It is possible to dig one step further looking at the relations (Eq. A.1) between the displacement jump $\llbracket U_{i} \rrbracket(X)$ and the stresses $\Sigma_{i j}(X)(i, j=X, Y, Z)$ along the cohesive zone, which involve the functions $\mathcal{A}_{p q}$ :

$$
\left\{\begin{array}{l}
\llbracket U_{X} \rrbracket(X)=0 \\
\llbracket U_{Y} \rrbracket(X)=\frac{4\left(1-v^{2}\right) d}{E}\left[\mathcal{A}_{22} \Sigma_{Y Y}(X)+\mathcal{A}_{12} \Sigma_{Z Z}(X)-\mathcal{A}_{23} \Sigma_{Y Z}(X)\right] \\
\llbracket U_{Z} \rrbracket(X)=\frac{4\left(1-v^{2}\right) d}{E}\left[-\mathcal{A}_{23} \Sigma_{Y Y}(X)-\mathcal{A}_{13} \Sigma_{Z Z}(X)+\mathcal{A}_{33} \Sigma_{Y Z}(X)\right] .
\end{array}\right.
$$

For $K_{I I I} / K_{I} \ll 1$, the $\mathrm{CZ}$ is predominantly in mode I and the main contribution of the $\mathrm{CZ}$ comes from $\llbracket U_{Y} \rrbracket(X) \sim$ $\frac{4\left(1-v^{2}\right) d}{E} \mathcal{A}_{22} \Sigma_{Y Y}(X)$, that is $\mathcal{A}_{22}(\alpha, \ell / d)$ whose values are multiply by a factor up to 50 when $\alpha=5^{\circ}$ and $\ell / d$ is changed from $\ell / d=1$ to $\ell / d=1.3$ (Fig. A.9).

\section{Appendix B. Machining of the notched specimens}

Ball-end end-mills Razor blade

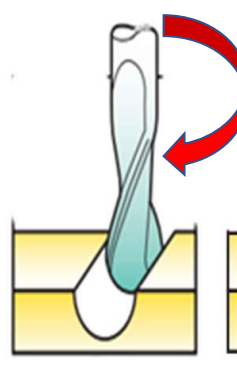

Notch

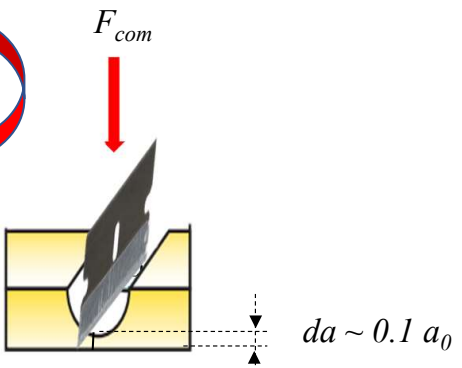

Sharp notch

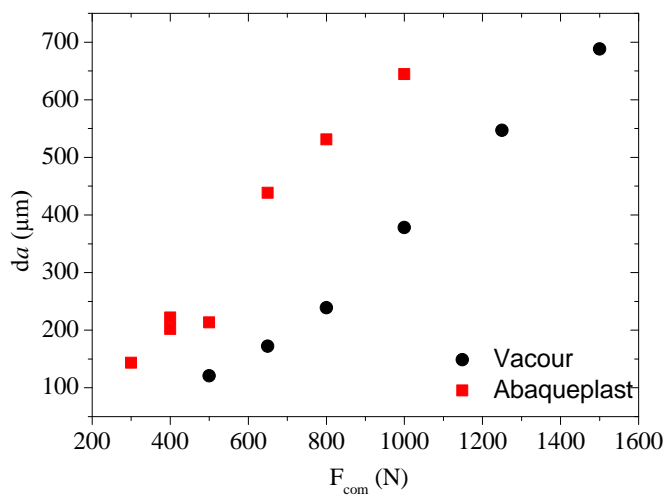

Figure B.10: Left: Notch milling and sharp notch formation. Right: Length of the additional precrack as a function of the compressive applied force for the two brands of PMMA (Vacour and Abaqueplast) used in this study.

Experiments are carried out using cast PMMA (with the common trade names of Plexiglass $($ Vacour) or Al-

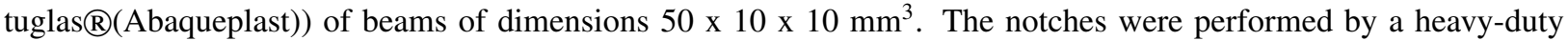
micro-milling machine (Roland MDX500) using a ball-end end-mills (HSS-E Cobalt from SURCO529) with a diameter of $400 \mu \mathrm{m}$. We chose different notch depths, $a_{0}=2,3$ and $4 \mathrm{~mm}$ with tilting angle, $\Gamma_{0}$ varying between $0^{\circ}$ (pure mode I) to $30^{\circ}$. As an end-mill is quite expensive and as drilling $a_{0}=3$ and $a_{0}=4 \mathrm{~mm}$ deep slits with such a 400 $\mu \mathrm{m}$ diameter mill necessitates frequent replacement because of the mill fragility, $a_{0}=2 \mathrm{~mm}$ was used for most of the samples.

The micro-milling was conducted under constant spindle rotational speeds $12000 \mathrm{rpm}$, an axial depths of cut 100 $\mu \mathrm{m}$ and a feed rate of $0.5 \mathrm{~mm} / \mathrm{s}$. At these conditions, there was no burr-formation. To initiate a true crack with a sharp tip and a smooth straight front, we pushed a wedge (razor blade) into the notch, quasi-statically, under displacementcontrolled condition at a constant rate of $0.1 \mathrm{~mm} / \mathrm{min}$, using an electro-mechanical testing machine (Instron 5882). The length of sharp crack is $d a \sim 0.1 a_{0}$ in every cases (Fig. B.10). The compressive force $F_{\text {com }}$ to apply to get a precrack lenght $\mathrm{d} a$ is given in Fig. B.10. The slit manufacturing introduces some residual stresses that are clearly visible as color fringes using two polarized sheets. After heating the samples at $90^{\circ} \mathrm{C}$ for 10 hours, we check that they 
have disappeared. The experimental protocol developed above permit rapid, accurate, efficient, and reproducible manufacturing of notch and sharp notch.

\section{Appendix C. Finite Element determination of the Stress Intensity Factors}

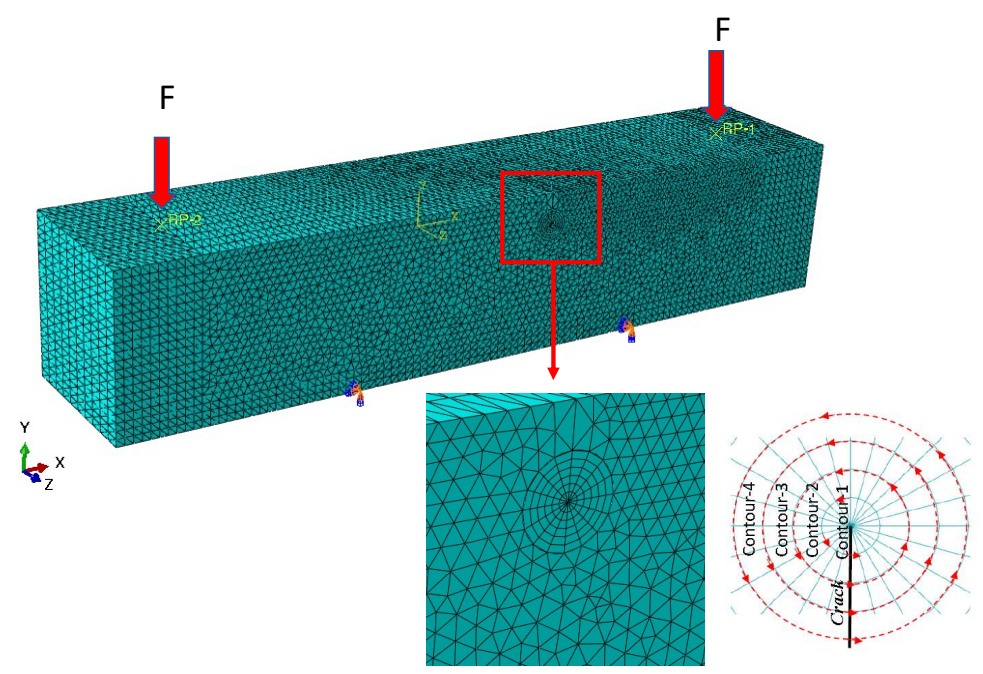

Figure C.11: Meshing used in the Finite Element model with the imposed Boundary Conditions

The Boundary Conditions are taken as follows (Fig. C.11): At the top, group of nodes defining the load lines are coupled to the rigid body motion defined by the reference nodes RP1 and RP2 and the force applied $F$ is distributed evenly; At the bottom, zero displacement along $Y$ is imposed along the contact lines. The meshes were constructed with C3D10: A 10-node quadratic tetrahedron, and a tetrahedral elements free meshing technique is assigned. The elasticity problem is solved using ABAQUS 6.14 environment (ABAQUS 2014) to get the displacement and stress fields. A spider web mesh around the crack tip is beneficial (Fig. C.11). To create such a mesh, a partition is made, corresponding to a circle centered on the crack tip. The spider web will be inside this circle.

To describe the $\sqrt{r}$ - behavior of the displacement field near the crack tip, mid-side nodes are moved towards the tip and hexahedral elements are collapsed to wedges with multiple nodes at each location at the crack tip. Provided that the user has defined the nodes of the crack front, the software automatically finds the contours in order to carry out the energetic analysis, and then returns the SIF values for each contour using the $J$-integral method. In our case, the near-tip mesh consists of 10 contours of elements meshed.

The values of $K_{I I} / K_{I}$ and $K_{I I I} / K_{I}$ along the crack front are given in Fig. C.12 for $a_{0}=2 \mathrm{~mm}$ and different values of $\Gamma_{0}$. As awaited near the edges (Bazant and Estenssoro, 1979; Dhondt et al., 2001), $K_{I I} / K_{I}$ becomes of the same order than $K_{I I I} / K_{I}$. However, in the center of the crack front, that is in the zone of interest for our study, $K_{I I I} / K_{I}$ is almost constant and predominant in comparison to $K_{I I} / K_{I}$. We choose to quantify the mode mixity by $K_{I I I} / K_{I} \equiv K_{I I I} / K_{I}(Z=0)$. The evolution of this quantity with $\Gamma_{0}$ is given in Fig. C.13.

Another output of the FE calculations is the displacement corresponding to the applied vertical forces. We checked that this displacement is nearly constant along the loading lines in agreement with our setup (Fig. 3a). From the compliance (ratio displacement/force) obtained by FE, the Young can be estimated from the loading curves. We get $E=2800 \mathrm{MPa}$ in agreement with the usual value of PMMA (Ashby, 1989). 

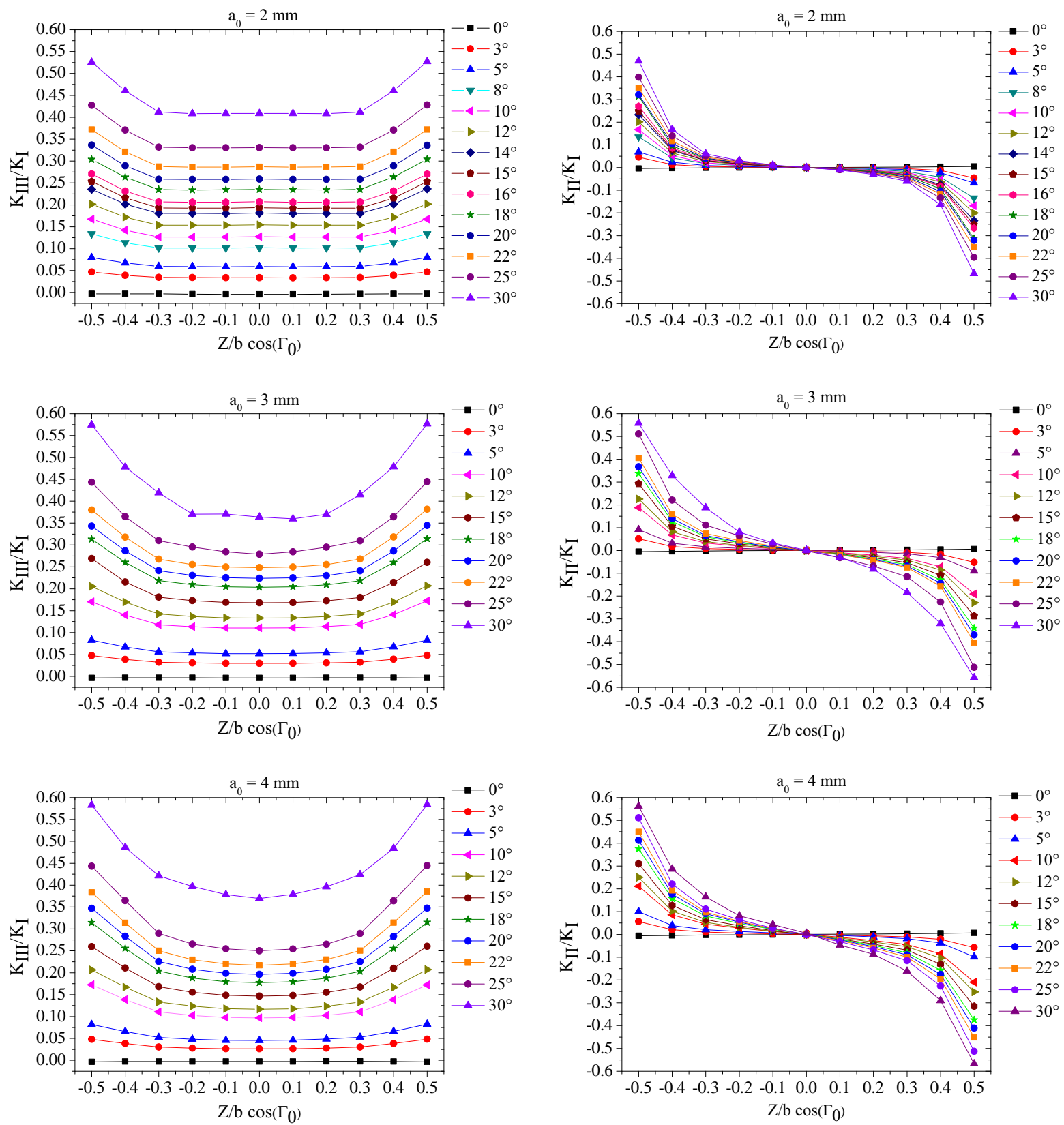

Figure C.12: Evolution of the ratio $K_{I I} / K_{I}$ and $K_{I I I} / K_{I}$ along the crack front as calculated by Finite Elements. 


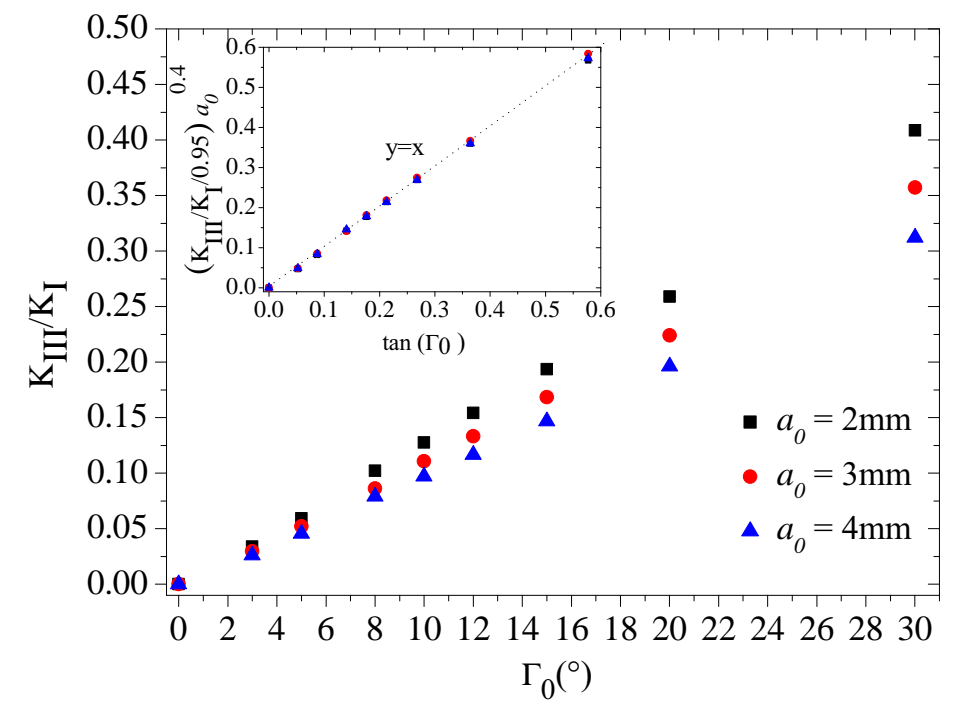

Figure C.13: Ratio of the mode III over the mode I SIF taken at the middle of the crack front. Inset: the dependance with $a_{0}$ and $\Gamma_{0}$ can be encompassed in the single formula (9). 